\title{
Low-velocity resistance distortion and bionic drag reduction for ship-type paddy field machinery
}

\author{
Guoqi Yan ${ }^{1}$, Peng Tian ${ }^{1}$, J iasi Mo ${ }^{1 *}$, Heng Xie ${ }^{1}$, Huiling Wei ${ }^{2}$ \\ (1. College of Engineering, South China Agricultural University, Guangzhou, 510642, China; \\ 2. College of Mechanical and Electrical Engineering, Foshan University, Foshan 528000, China)
}

\begin{abstract}
The resistance between mud surface and ship-type paddy field machine hull accounts for a certain proportion of the total mechanical power consumption during the operation process. The special characteristic has been found via preliminary study, it showed that when starting or working at low velocity, the resistance is much greater than the machine working at the rated velocity. This characteristic affects the dynamic matching and structural design of the machine. The critical velocity of resistance distortion was around $0.025 \mathrm{~m} / \mathrm{s}$ under the setting experimental conditions, and the mechanism of resistance distortion under multi-medium was analyzed. Based on water film drag reduction theory, a macroscopic structure of crocodile's abdominal armor biomimetic drag reduction method was proposed. Two kinds of bionic macroscopic structure ship board were fabricated. The macroscopic structure can introduce more water into the bottom surface of the hull; thicken the water film, which can reduce the travel resistance. Design and manufacture a ship-type paddy field experimental setup with velocity control, and compare the traditional smooth surface ship board with the two bionic macroscopic structure surfaces through orthogonal test. The experiment results demonstrated that the ship board with rectangle gully structure has better drag reduction effect than the hexagon one in the lower velocity. But when the travel velocity approach to the resistance distortion critical point, the hexagon gully structure become better than the other one. Through the orthogonal test, the best drag reduction combination is $2.5 \mathrm{~kg}$ load weight, $0.025 \mathrm{~m} / \mathrm{s}$ travel velocity with hexagon ship board, which decreases the drag by $6.3 \%$.
\end{abstract}

Keywords: ship-type paddy field machinery, low velocity, resistance distortion, bionic, drag reduction, crocodile DOI: $10.25165 /$ j.ijabe.20201302.5417

Citation: Yan G Q, Tian P, Mo J S, Xie H, Wei H L. Low-velocity resistance distortion and bionic drag reduction for ship-type paddy field machinery. Int J Agric \& Biol Eng, 2020; 13(2): 7-14.

\section{Introduction}

Rice is one of the main food crops in China, mainly based on paddy field cultivation. The ship-type paddy field operation machinery occupies a certain proportion in small and medium-sized paddy field machinery ${ }^{[1]}$. During the operation, the ship-type paddy field mechanical ship board is the main load-bearing structure. The viscous resistance between paddy field mud and bottom of ship hull is one of the main factors which affecting the working efficiency of the ship-type paddy field machinery. The resistance between ship board and the soil during the operation of ship-type paddy field machinery accounts for the total energy consumption about $20 \%$ to $40 \%{ }^{[2]}$. In the previous research, it was found that the resistance between the ship board and the mud surface is much higher than the resistance at the rated velocity when the ship-type paddy field machinery is at the starting state or

Received date: 2019-09-22 Accepted date: 2020-02-13

Biographies: Guoqi Yan, Doctoral degree, Associate Professor, research interests: agricultural equipment research, Email: ygq1978@scau.edu.cn; Peng Tian, Master, research interests: agricultural machinery research, Email: 309769546@qq.com; Heng Xie, PhD, Lecturer, research interests: biomimetic machinery research, Email: xieheng@scau.edu.cn; Huiling Wei, PhD, Lecturer, research interests: mechanical design research, Email: weihuiling2007@ 126.com.

*Corresponding author: Jiasi Mo, PhD, Lecturer, research interests: agricultural robot research. College of Engineering, South China Agricultural University, Guangzhou 510642, China. Tel: +86-20-85280868, Email: mo_jiasi@scau.edu.cn. the lower velocity running state. The resistance characteristic of this distortion is different from the general conditions. It seriously affects the design and applications for power matching of ship-type paddy field machinery driven by motors ${ }^{[3]}$.

Bionic drag reduction technology has more mature applications in agricultural machinery, aircraft and ship engineering ${ }^{[4-7]}$. For example, the colter boot and plough designed by the non-smooth surface structure of the bionic scorpion surface ${ }^{[8,9]}$, the bulldozer designed with the non-smooth surface structure of the bionic scorpion head ${ }^{[10]}$, the bionic swimsuit which imitates the micro-structure design of shark $\operatorname{skin}^{[11]}$, and so on ${ }^{[12-14]}$. These cases have a good drag reduction effect. In the past, the study of bionic drag reduction mainly focused on the study of drag reduction methods in the case of single medium or high-velocity motion. And the micro structure design is the mainly method for drag reduction. Micro structure drag reduction is based on wettability. Wettability is the process by which one liquid replaces another immiscible solution on a solid surface. In this paper, a drag reduction method for the velocity range of resistance distortion and distortion interval in paddy field surface including water layer, mud-water mixture layer and hard mud layer and the interaction between state and bottom of ship board is studied. The mud-water mixture consists of water layer, mud-water layer, mud layer, and some large particles, so the wettability is not a suitable way to describe the phenomenon of this paper, so does the traditional Cassie model. At present, there is no suitable mathematical model to describe the mud-water mixture of the experiment environment in application of ship-type paddy field 
machinery. Therefore, experimental research method is adopted to summarize the law. On the other hand, using micro structures to achieve wettability drag reduction is expensive; it is not a suitable method for agricultural machinery. Based on the current situation of popularization and application of agricultural machinery and its technology in China, cost is the key factor. In this paper, a macroscopic structure drag reduction method which suitable for ship-type paddy field machinery was proposed, so as to reduce processing difficulty and production cost, so that the research results of this paper can be more easily accepted by farmers and used in agricultural practice. If the macroscopic structure can achieve a certain drag reduction effect, its cost will be greatly reduced, and it can be better promote to the agricultural machinery application. Therefore, macroscopic structure drag reduction method is adopted instead of the micro structures in this paper.

In order to meet the special requirements of the research object, small scale paddy field experimental equipment with precise velocity control was developed. According to the ship board resistance distortion characteristics and drag reduction mechanism analysis, the non-smooth ship board surface macroscopic structure was designed from the bionic crocodile armor. The drag reduction effect of ordinary smooth ship board is compared with the bionic ship board by experiment.

\section{Resistance distortion characteristic experiment}

\subsection{Introduction of experimental setup}

The actual paddy field environment is complex and the experiment workload is large. In order to obtain reliable experiment data, ensure the stability of the repetitive experiment environment conditions and improve the experiment efficiency, small paddy field experimental equipment is designed and manufactured. The schematic diagram of the principle is shown as Figure 1.
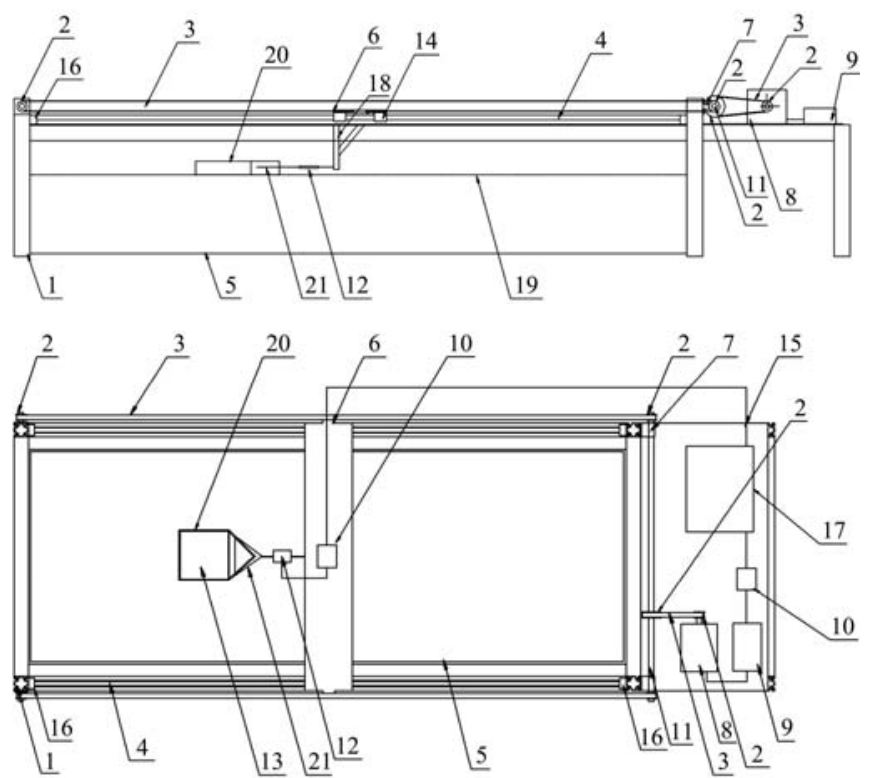

1. Bench 2. Synchronous wheel 3. Synchronous belt 4. Linear guideway 5. Tank structure 6. Traction table 7. Guideway support 8 . Motor 9. Inverter 10. Transmitter 11. Linear guideway 12. Tension sensor 13. Ship board 14. Slider 15. Operating platform 16.Limit 17. Computer 18. Traction frame 19. Mud surface 20. Ship hull 21. Ship board traction position

Figure 1 Design diagram of the experimental setup

The small paddy field experimental equipment consists of a soil tank, a variable frequency velocity control system, a traction system, a measurement system and a control system. The actual paddy field environment is simulated in the soil tank. The variable frequency velocity control system is mainly composed of an AC motor with mechanical reducer and an inverter, providing a precise and controllable velocity range. The traction system travels on the mud surface by a vertical arm and a tension sensor to pull the ship board, and the traction direction is parallel to the traveling direction and close to the resistance action surface. Since the experiment setting is carried out at a lower velocity, the actual velocity of the ship board is uniform, calculated from the travel distance and time, and the resistance data is measured by the tension sensor. The actual experimental setup is shown in Figure 2.

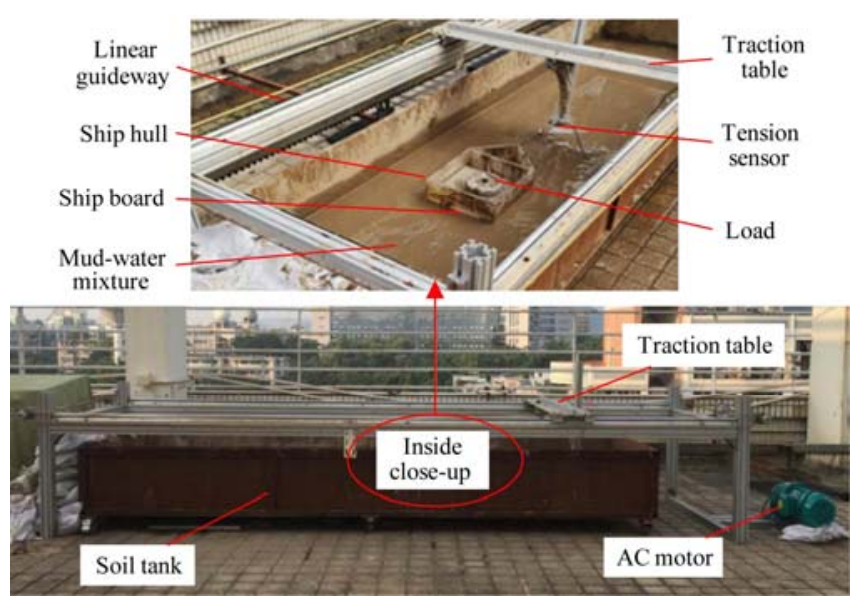

Figure 2 The manufactured experimental setup

According to the soil environment characteristics of paddy fields in Southern China ${ }^{[15]}$, the average paddy soil with a depth of $20 \mathrm{~cm}$ is placed in the soil tank, and the average depth of the water layer is $3 \mathrm{~cm}$. The paddy soil is screened and does not contain impurities such as large particles and plant root residues. Because every traction test will change the path of the ship board mud surface conditions, in order to ensure the consistency of the experiment conditions and the reliability of the experiment data, the mud surface in the soil tank have be deep turned and leveled for more than $10 \mathrm{~h}$ in the first experiment. The same parameter experiment was conducted continuously for more than 5 times. Before each experiment, the mud surface was levelled before each test and the interval was $10 \mathrm{~min}$. The experiment data of the first time and the maximum deviation were removed; the remaining sets of experiment data will be averaged and take as the valid experiment data. The mud surface in the soil tank was flattened again and then sits for more than $10 \mathrm{~h}$; another set of parameters was experimented.

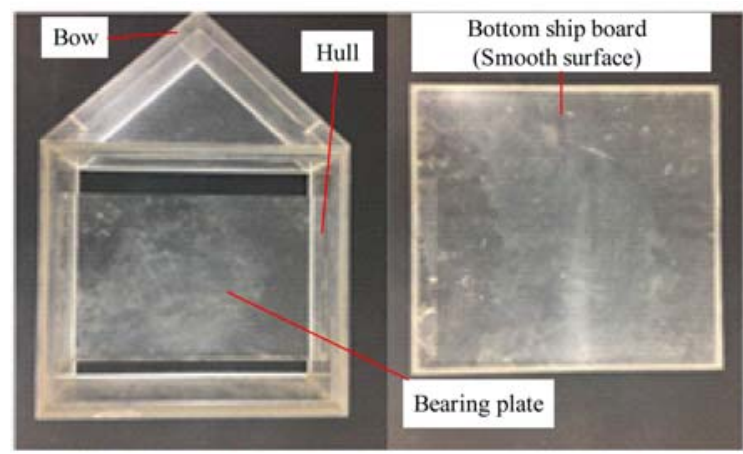

Figure 3 Experiment ship hull structure

The design scheme of modularization experiment ship board is adopted, which consists of hull and bottom board. The bottom board can be embedded in the bottom groove of the hull to 
facilitate the replacement of the bottom board of different specifications. In order to reduce the impact on the experiment data which come from the irregular mud accumulation in the ship bow. The bow of the hull is designed as a triangle. The load-bearing plate was installed fixed in the middle of the hull to place different load weight. In order to facilitate the observation of the interaction between the mud surface and the bottom ship board surface during experiment, the experiment ship board is made entirely of transparent material, and the dimension of the bottom ship board is $300 \mathrm{~mm} \times 300 \mathrm{~mm} \times 15 \mathrm{~mm}$. The structure of the experiment ship board is shown in Figure 3.

\subsection{Analysis of experimental data}

In order to explore the resistance distortion characteristic experiment, the ordinary smooth bottom board is selected as the experiment object. In the velocity range from 0.005 to $0.050 \mathrm{~m} / \mathrm{s}$, a group of experiments were conducted at intervals of $0.005 \mathrm{~m} / \mathrm{s}$, and there were 10 velocity experiment points. The load is $2.5 \mathrm{~kg}$. The experiment results are described in Table 1.

Table 1 Relationship between velocity and pull

\begin{tabular}{cc||cc}
\hline Velocity $/ \mathrm{m} \cdot \mathrm{s}^{-1}$ & Pull/N & Velocity $/ \mathrm{m}^{-1} \mathrm{~s}^{-1}$ & Pull/N \\
\hline 0.005 & 30.6 & 0.030 & 17.5 \\
0.010 & 22.2 & 0.035 & 20.6 \\
0.015 & 17.4 & 0.040 & 22.0 \\
0.020 & 16.9 & 0.045 & 22.9 \\
0.025 & 16.0 & 0.050 & 24.2 \\
\hline
\end{tabular}

According to the data in Table 1, the ship board resistance fitting curve is drawn as shown in Figure 4.

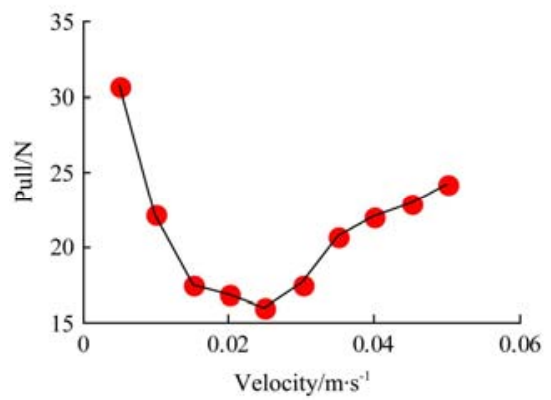

Figure 4 Resistance fitting curve

It is observed from Figure 4 that the critical point of the resistance distortion occurs at a velocity of around $0.025 \mathrm{~m} / \mathrm{s}$ under the setting experiment conditions. When the velocity is greater than $0.025 \mathrm{~m} / \mathrm{s}$, the ship resistance is positively correlated with the velocity, which accords with the general law of viscous resistance. When the velocity is lower than $0.025 \mathrm{~m} / \mathrm{s}$, the resistance of the ship board increases with the decrease of the velocity, and distortion happens.

The data of Table 1 is subjected to polynomial interpolation fitting equation. The relationship between ship velocity and ship board resistance is as follows:

$$
\begin{aligned}
& y=4.39 \times 10^{16} x^{9}-1.26 \times 10^{16} x^{8}+1.53 \times 10^{15} x^{7}-1.04 \times 10^{14} x^{6}+ \\
& 4.26 \times 10^{12} x^{5}-1.1 \times 10^{11} x^{4}+1.74 \times 10^{9} x^{3}-1.61 \times 10^{7} x^{2}+ \\
& 7.69 \times 10^{4} x-110.2
\end{aligned}
$$$$
\text { where, } y \text { is the ship board resistance, } x \text { is the ship board velocity. }
$$

\section{Bionic ship board design and experiment}

\subsection{Drag reduction mechanism of macroscopic structure}

The experiment paddy soil can be divided into three kinds of medium layers: water layer, mud-water mixed layer and hard mud layer from top to bottom. During the experiment, through the transparent hull observation, when the hull is standing on the mud surface, under the load of gravity, the mixed layer of water layer and muddy water was squeezed out by the bottom of the hull, and the ship board surface will be tightly contact to the mud. There was only a thin water film ${ }^{[16]}$. When the ship board is traveling at a lower velocity under the resistance distortion critical point, the water film is consumed, and the ship board is not enough to feed the water layer in front of the ship board. At the same time, the water layer on the side of the ship board cannot effectively enter between the bottom board and the mud surface. The hull bottom board and the mud surface are in a tightly viscous state, resulting in greater resistance. The resistance is mainly caused by the gravity between the hull bottom and the soil molecules, and the capillary force generated by the water meniscus when the water infiltrates the soil and the ship board ${ }^{[17]}$. The production of water film between the bottom of the hull and the mud surface can directly contact the soil and the bottom board to reduce the molecular attraction of the soil. When the travel velocity is high, the water layer in front of the ship board is compensate in time, and the mixed layer of water layer and mud water is not enough to be completely discharged by the bottom board of the hull. During the whole process, a certain thickness of water is moderately maintained between the bottom board and the mud surface. The water film reduces the tension of the water molecules and reduces the capillary force ${ }^{[18]}$, and the resistance is significantly reduced. Therefore, water film thickness is the key factor affecting the resistance of the ship board at lower velocity.

The resistance distortion can be explained by the water film theory, so it can also use for drag reduction. The water film theory is a classical model of soil and solid viscosity ${ }^{[19]}$. It points out that the adhesion between the soils and foreign materials can be attributed entirely to the water film between the joined surfaces; And within the 'sticky' range at any move rate, the value of the adhesion was found to be equal to the product of the area of the water film joining the two surfaces and the tension within it. Another further research found that the larger water film area and thinner water film will make greater viscous force ${ }^{[20]}$, as shown in Figure 5. The ship-type paddy field machinery is in full contact with the mud-water mixture, so it is difficult to change its water film area, but it is possible to introduce more water into the surface, so the thickness of the water film will increase, making the viscous force decreases and reduce the drag. The key of drag reduction is to find a suitable macroscopic structure on the bottom surface of the hull (ship board).

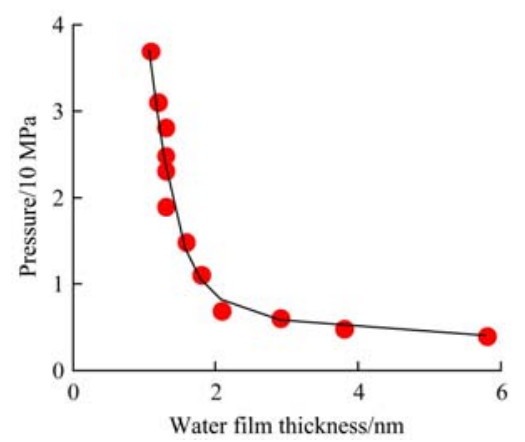

Figure 5 Water film thickness vs the separation pressure ${ }^{[20]}$

If the ship board has a certain connecting gully structure, a part of water will remain in the groove when the bottom board is in contact with the mud surface. During the traveling of the ship 
board, the water inside the gully can be quickly moved to the ship board to thicken the water film ${ }^{[21]}$. Due to the negative pressure created by the moisture consumption in the gully, water can be replenished through the openings on both sides of the board to maintain a continuous water film throughout the travel, reducing the resistance between the ship board and the mud. A drag reduction mechanism diagram based on water film theory is shown in Figure 6.

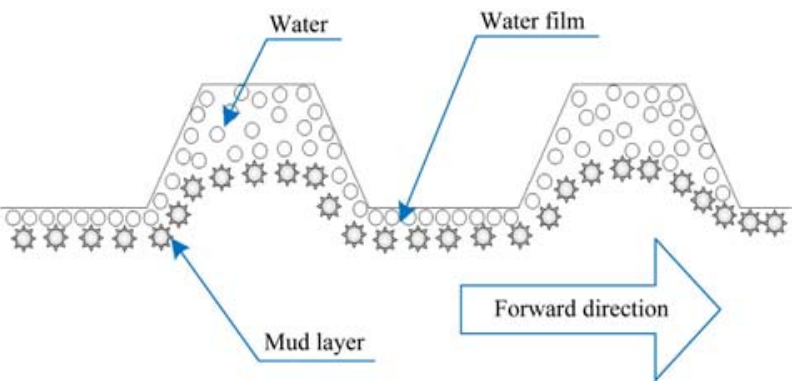

Figure 6 Drag reduction mechanism diagram based on water film theory

\subsection{Bionic prototype selection}

Because the complex of paddy field environment and considering that agricultural machinery require low-cost, in order to reduce processing difficulty and production cost, so chose the macroscopic drag reduction structure instead of micro structure.

The design basis of macroscopic structure is mainly from the bionics, looking for some living creatures in the mud-water mixture environment (swamp), and its appearance has macroscopic structure. It is easy to associate with amphibious reptiles such as crocodiles that the abdominal armor structure has macroscopic gully that can introduce water and thicken the water film. And the crocodile's abdominal armor has a macroscopic structure (rectangle), as shown in Figure 7, which is not the same shape as the scales in other parts, as show in Figure 8.

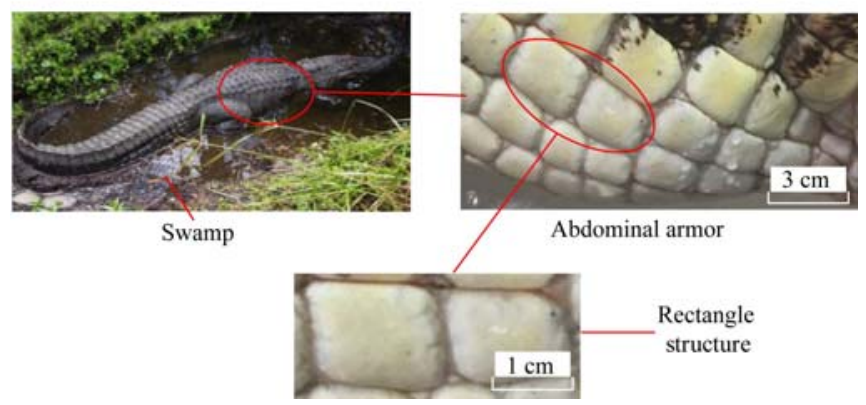

Figure 7 Crocodile’s abdominal armor structure

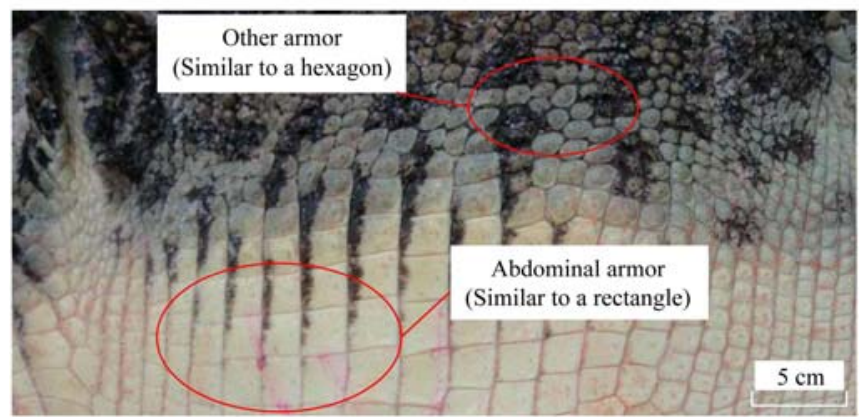

Figure 8 Compare the structures of other armor with abdominal armor

Crocodiles were selected as bionic prototype. The vast majority of crocodile living environment is close to the paddy field environment. Under normal circumstances, the crocodile is move slow and stays in the swamp for a long time. When crawling, the abdomen and the mud surface are often in contact, and the abdominal armor has drag reduction characteristics.

It was observed that the crocodile lower abdomen and the lower part of the extremities were distributed with a relatively uniform convex hull structure, and the convex hulls were connected through the grooves. The convex bulge shape of the lower abdominal armor is alternately arranged in a shape of a rectangle, and the convex hull shape of the lower armor of the extremities is a staggered arrangement of hexagon. The structure size of the abdominal armor was measured. The width of the groove was about $1 / 3-1 / 10$ of the width of the convex hull. The depth of the groove was about 1-3 times of the width of the groove.

\subsection{Bionic ship board design}

According to the bionic study on the macroscopic structure of the crocodile, this paper designed two macroscopic bionic structures based on the crocodile's armor, one is rectangle, and another is hexagon. These two shapes are designed through the statistics of the crocodile's armor shape characteristics, and the unification of the normalization, as well as scale up, so it can reduce the processing cost and difficulty. Designed these two simplify shapes of the crocodile's armor is used for compared, so the better shape for drag reduction will be find out through experiment.

Acrylic material is not an ideal material for drag reduction, but because of the transparency, it is a better way for observing the water film changing through the whole process of the experiment (according to the drag reduction mechanism based on water film theory, water film thickness is the key parameter to the drag reduction), it is good for the experiment analysis, so the transparent acrylic material is chosen for build the experiment setup. In addition, this paper studies the effect of macroscopic structure on drag reduction, not the effect of materials on drag reduction. Therefore, as long as each group of experimental materials is consistent, the experimental results are comparable and valid. The ship board dimension is $300 \mathrm{~mm} \times 300 \mathrm{~mm} \times 25 \mathrm{~mm}$. The experiment scheme of Section 2.1 is adopted.

The design of two bionic structure bottom boards and the connecting groove structure are shown in Figure 9. The groove cross section is trapezoidal, the groove depth is $15 \mathrm{~mm}$, the groove width is $10 \mathrm{~mm}$, the groove bottom width is $3 \mathrm{~mm}$, and the two groove spacing is $50 \mathrm{~mm}$. The two bionic ship boards have the same dimension, and the manufactured bionic ship board is shown in Figure 10.
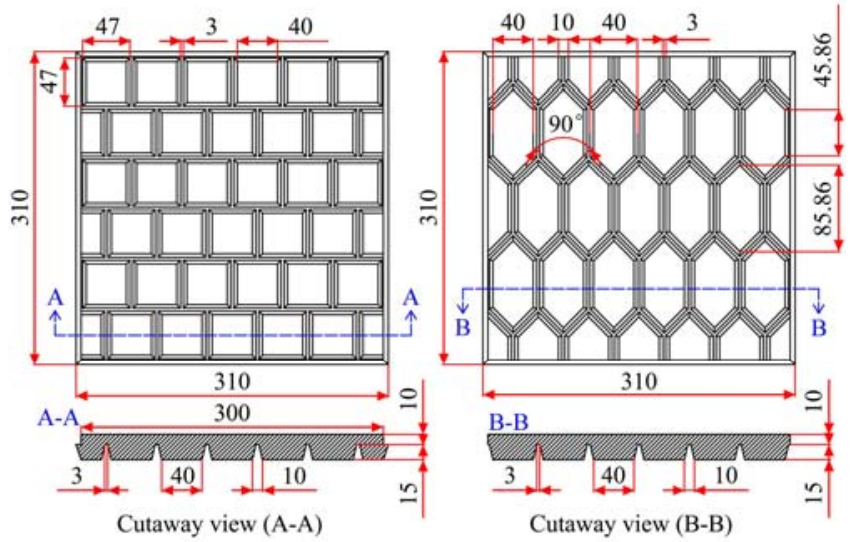

Figure 9 Design diagram of bionic structure 


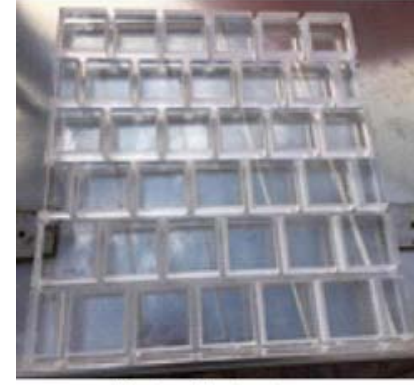

Rectangle structure

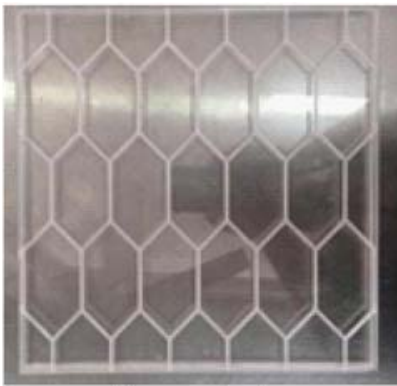

Hexagon structure
Figure 10 Two kinds of bionic ship board

\section{Orthogonal experiment and data analysis}

\subsection{Experiment design}

The purpose of the experiment is to verify the drag reduction effect by measure the resistance of the two bionic ship boards and compare with smooth surface ship board under the same experiment conditions through the experimental setup which introduced in section 2. The variable factors of the ship board resistance are travel velocity, load weight and the ship board structure, which can constitute a three-factor and three-levels experiment plan.

The goal of the orthogonal test is to find the better combination under lesser tests. For example, a three-factor, three-level test should be conducted. According to the requirement of comprehensive test, the $3 \times 3 \times 3=27$ combinations should be carried out through the tests, and the number of repetitions of each combination has not been considered. If the test is arranged according to $\mathrm{L} 9\left(3^{4}\right)$ orthogonal table, only 9 tests are needed, obviously the workload is greatly reduced. Therefore, the work done in this plan is sufficient.

This paper mainly studies the resistance distortion problem and the drag reduction method in the distortion range. In this section, the experiment travel velocity is designed below $0.025 \mathrm{~m} / \mathrm{s}$ (resistance distortion critical point), so the experiment points are set to $0.015 \mathrm{~m} / \mathrm{s}, 0.020 \mathrm{~m} / \mathrm{s}$ and $0.025 \mathrm{~m} / \mathrm{s}$.

The ship load weight affects a lot on the resistance, and the ship board pressure is calculated as follows;

$$
P=\frac{G}{B L}
$$

where, $P$ is the grounding pressure; $G$ is the gravity of the ship board; $B$ is the grounding width of the ship board; $L$ is the grounding length of the ship board.

Taking a small hand-held transplanter as an example, the effective mass range of the ship board is between 20 to $35 \mathrm{~kg}$, the effective grounding length is $80-100 \mathrm{~cm}$, and the grounding width is $40 \mathrm{~cm}$ (double ship board). According to Equation (2), the mass of the experiment ship board is about $6.0 \mathrm{~kg}$, so the experiment load weights are set to $2.5 \mathrm{~kg}, 5.0 \mathrm{~kg}$ and $7.5 \mathrm{~kg}$, respectively, which is matching with the actual situation. The orthogonal experiment factors and levels are shown in Table 2.

Table 2 Orthogonal test factors and level

\begin{tabular}{cccc}
\hline & \multicolumn{3}{c}{ Factors } \\
\cline { 2 - 4 } Level & A: & B: & C: \\
& Travel velocity $/ \mathrm{m}^{-1}{ }^{-1}$ & Load weight $/ \mathrm{kg}$ & Ship board structure \\
\hline 1 & 0.015 & 2.5 & Smooth surface \\
2 & 0.020 & 5.0 & Rectangle structure \\
3 & 0.025 & 7.5 & Hexagon structure \\
\hline
\end{tabular}

\subsection{Resistance comparison experiment}

For the three factors and three level test, the $\mathrm{L} 9\left(3^{4}\right)$ orthogonal table can be selected, and the test plan and results are shown in Table 3. The test results in the table are analyzed by the method of intuitive analysis. Intuitive analysis requires the calculation of the range $R$ of each factor, by comparing the magnitude of range $R$ between the factors, the influence degree of each factor was ranked. The calculation method of the range $R$ is as follows. Firstly, the sliding resistance values of all factors at the same level are summed and calculated, as,

$$
K_{i}=\sum_{i=i}^{s} y_{i} \# \# \# \# \# \# \# \# \# \# \# \# \# \# \#
$$

where, $y_{i}$ is the sliding resistance value of a factor of level $i$; $s$ is the number of trials at the same level. $K_{\mathrm{i}}$ is calculated as follows

$$
k_{i}=K_{i} / s
$$

The formula for the range $R$ is

$$
\begin{array}{r}
\text { or } \quad R=\max \left\{K_{1}, K_{2}, \ldots, K_{i}\right\}-\min \left\{K_{1}, K_{2}, \ldots, K_{i}\right\} \\
R=\max \left\{k_{1}, k_{2}, \ldots k_{i}\right\}-\min \left\{k_{1}, k_{2}, \ldots, k_{i}\right\}
\end{array}
$$

This experiment uses Equation (6) to calculate the extreme difference of each factor. According to the formula, the values of

\begin{tabular}{|c|c|c|c|c|c|}
\hline \multirow{2}{*}{$\begin{array}{c}\text { Experiment } \\
\text { number }\end{array}$} & \multicolumn{4}{|c|}{ Factors } & \multirow{2}{*}{$\begin{array}{c}\text { Resistance } \\
\text { /N }\end{array}$} \\
\hline & $\begin{array}{l}\text { A: Travel } \\
\text { velocity }\end{array}$ & $\begin{array}{l}\text { B: Load } \\
\text { weight }\end{array}$ & $\begin{array}{l}\text { C: Ship board } \\
\text { structure }\end{array}$ & $\begin{array}{l}\text { D (Empty } \\
\text { column) }\end{array}$ & \\
\hline 1 & 1 & 1 & 1 & 1 & 21.31 \\
\hline 2 & 1 & 2 & 2 & 2 & 22.11 \\
\hline 3 & 1 & 3 & 3 & 3 & 30.17 \\
\hline 4 & 2 & 1 & 2 & 3 & 17.33 \\
\hline 5 & 2 & 2 & 3 & 1 & 18.77 \\
\hline 6 & 2 & 3 & 1 & 2 & 26.57 \\
\hline 7 & 3 & 1 & 3 & 2 & 13.22 \\
\hline 8 & 3 & 2 & 1 & 3 & 17.4 \\
\hline 9 & 3 & 3 & 2 & 1 & 23.22 \\
\hline$K_{1}$ & 73.59 & 51.86 & 65.28 & 63.3 & I \\
\hline$K_{2}$ & 62.67 & 58.28 & 62.66 & 61.9 & I \\
\hline$K_{3}$ & 53.84 & 79.96 & 62.16 & 64.9 & I \\
\hline$k_{1}$ & 24.53 & 17.29 & 21.76 & 21.1 & I \\
\hline$k_{2}$ & 20.89 & 19.43 & 20.89 & 20.63 & I \\
\hline$k_{3}$ & 17.9 & 26.65 & 20.72 & 21.63 & I \\
\hline Range $R$ & 6.63 & 9.36 & 1.04 & 1 & I \\
\hline Factor order & & & $\mathrm{B}>\mathrm{A}>\mathrm{C}>\mathrm{D}$ & & \\
\hline $\begin{array}{c}\text { Optimal } \\
\text { combination }\end{array}$ & & & $\mathrm{A}_{3} \mathrm{~B}_{1} \mathrm{C}_{3} \mathrm{D}_{2}$ & & \\
\hline
\end{tabular}
$K_{i}, k_{i}$ and $R$ are calculated, the orthogonal test results of the ship board travel resistance are analyzed, and the influence degree of the three factors is compared by calculating the range $R$ of different factors.

Table 3 Orthogonal experiment plan and results

As can be seen from Table 3, the range of the factor $A$ is 6.63 , the range of the factor $\mathrm{B}$ is 9.36, the range of the factor $\mathrm{C}$ is 1.04 , and the range of the factor $\mathrm{D}$ is 1.00. Through the range comparison, it can be seen that the load weight factor will influence the drag greater than the travel velocity factor, and the ship board structure factor has the least influence on the drag. When the travel velocity is $0.025 \mathrm{~m} / \mathrm{s}$, the ship load weight is $2.5 \mathrm{~kg}$, and the ship board structure is hexagon, has the best drag reduction effect, so the optimal combination is $\mathrm{A}_{3} \mathrm{~B}_{1} \mathrm{C}_{3} \mathrm{D}_{2}$. 
In order to further verify the experiment results, a systematical experiment was carried out on the bionic ship board. The combined experiments of different load weight and travel velocity were carried out for three different structures of ship board. In order to reduce the experiment error, the experiment method of section 2.1 is used, and the three experiment data is taken to calculate the average value. The experiment data is shown in Table 4.
The drag reduction effect in Table 4 is the ratio of the measured resistance difference between the bionic ship board and the smooth surface ship board under the same experimental conditions. The positive sign of the last column in Table 4 indicated the drag is decrease, and the negative sign indicated the drag is increase. It can be seen that the two kinds of bionic ship board has a certain drag reduction effect.

Table 4 Statistics of combined test data

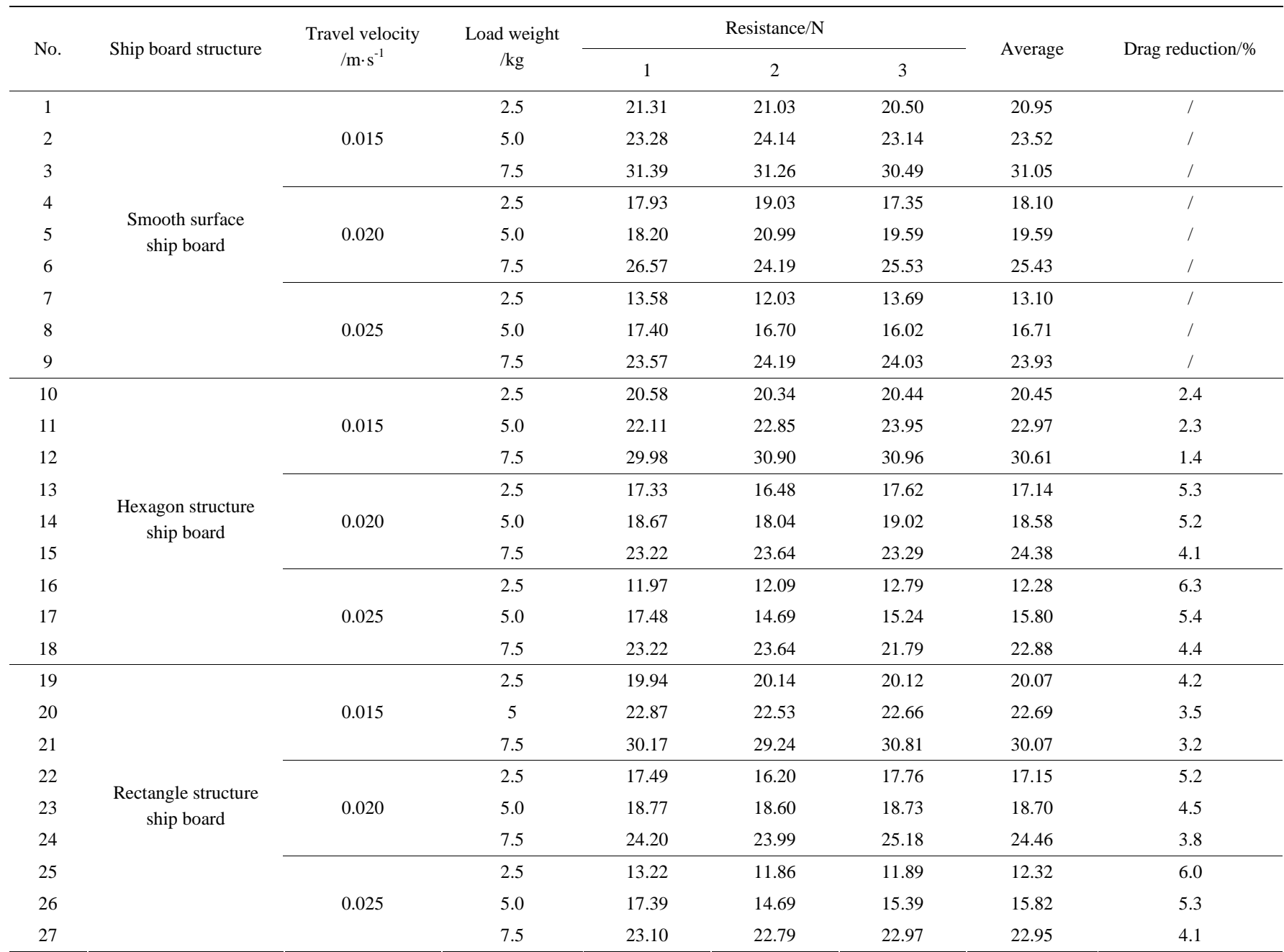

\subsection{Experiment results analysis}

Statistical analysis was performed from the data in Table 4, there are two comparisons will be taken, one is keeping load weight constant and compare drag reduction effect at different velocity (Figures 11-13); another is keeping velocity constant and compare drag reduction effect at different load weight (Figures 14-16).

It can be seen from the above figure that the travel resistance of different ship boards are significantly affected by the load weight. When the ship board structure and the load weight are specific, the travel resistance in low travel velocity present the uniform law described in section 2.2, when travel velocity under the resistance distortion critical point, the drag decreases as the velocity increases. When the travel velocity and the load weight are not change, the bionic ship board has a certain drag reduction effect; the drag of the two bionic ship boards is less than the smooth surface ship board. The data shows that the better combination is $2.5 \mathrm{~kg}$ load weight, $0.025 \mathrm{~m} / \mathrm{s}$ travel velocity, and the bionic hexagon structure ship board, the drag reduction effect is
$6.3 \%$.

The travel velocity in the better drag reduction combination is around the resistance distortion critical point, and the drag reduction effect of the two bionic ship boards are almost the same. But if the velocity is much lower than the resistance distortion critical point, such as the $0.015 \mathrm{~m} / \mathrm{s}$ in this experiment, the difference of the two bionic ship boards will tell, as show in Figures 14-16.

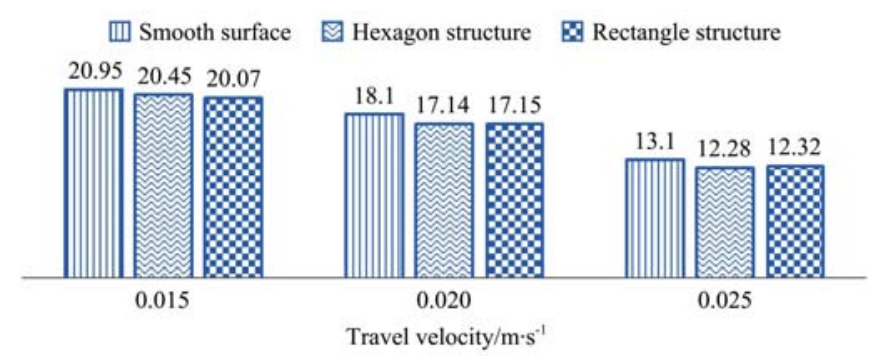

Figure 11 Travel resistance with 2.5kg Load weight 
四 Smooth surface $\otimes$ Hexagon structure 8 Rectangle structure

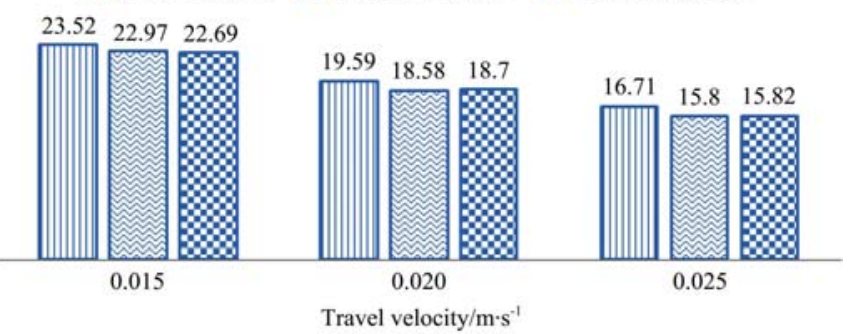

Figure 12 Travel resistance with $5.0 \mathrm{~kg}$ Load weight

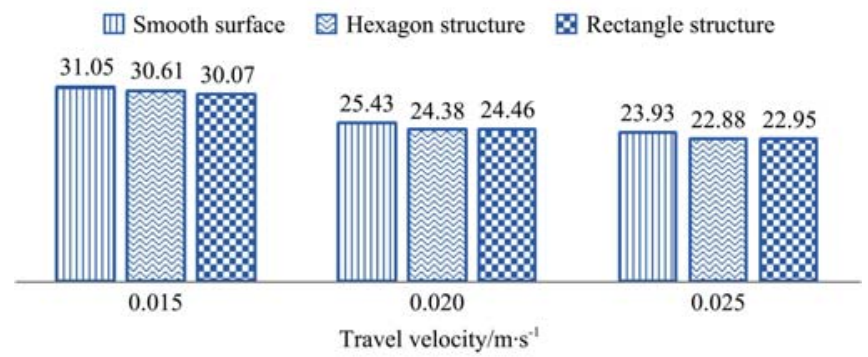

Figure 13 Travel resistance with $7.5 \mathrm{~kg}$ Load weight

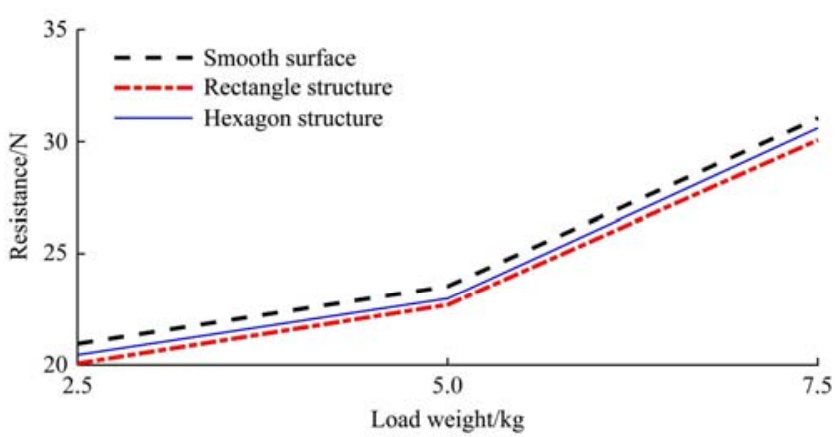

Figure 14 Travel resistance with $0.015 \mathrm{~m} / \mathrm{s}$ velocity

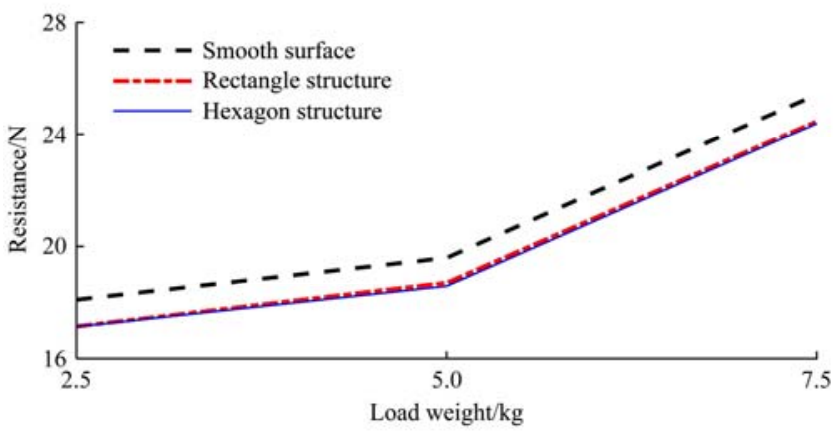

Figure 15 Travel resistance with $0.020 \mathrm{~m} / \mathrm{s}$ velocity

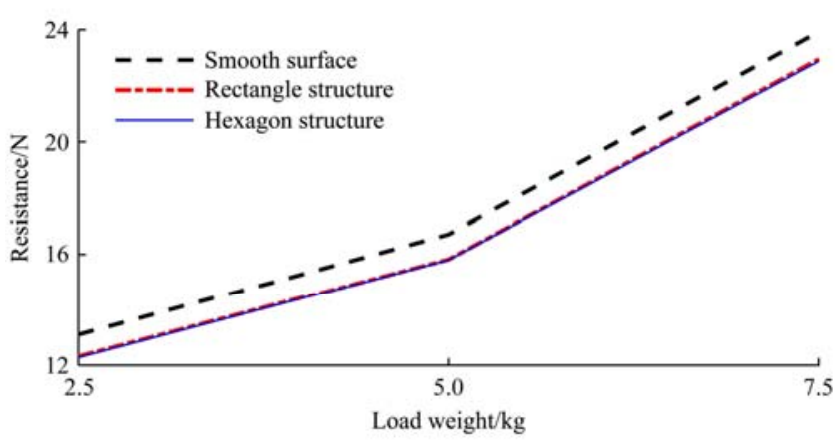

Figure 16 Travel resistance with velocity of $0.025 \mathrm{~m} / \mathrm{s}$

As shown in the Figure 14, when the ship board travels in a lower velocity, the resistance is obviously, and the rectangle structure has a much better drag reduction effect than the others. When the travel velocity increase, and approaching to the resistance distortion critical point, the drag reduction effect of the two bionic structure ship boards are getting closer and closer.

The experimental results are consistent with the drag reduction mechanism proposed in section 3.1. The thickness of water film is the key to reduce drag of ship-type paddy field machinery, if the macroscopic structure can increase the thickness of water film, a certain drag reduction effect can be achieved. The experiment shows that the rectangle structure similar to the crocodile's abdominal armor could maintain the thickness of the water film, achieving a better drag reduction effect in lower velocity. Further observe of other amphibious reptiles, such as turtles. From the shape of the armor on the turtle's abdomen and back, it can be seen that the armor on the turtle's abdomen also evolved into a rectangle shape of structure, which is consistent with the experimental results. The rectangle structure has a better drag reduction effect in lower velocity, and when the velocity increase, the hexagon structure has better and better drag reduction effect. Therefore, the size parameters of the gully structure optimization for better effect of drag reduction will be study subsequently, so does the ship board materials.

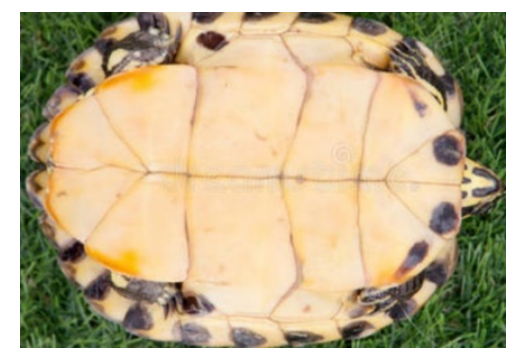

Figure 17 Rectangle shape of structure on turtle's abdominal armor

\section{Conclusions and prospect}

The resistance distortion characteristics experiment in this paper reveals the relationship between resistance and velocity of the ship board in the multi-medium environment at low velocity, the resistance distortion critical point is around $0.025 \mathrm{~m} / \mathrm{s}$. The orthogonal experiment shows that the bionic design of ship board has a certain drag reduction effect in the resistance distortion range. And the ship board with rectangle macroscopic structure has better drag reduction effect in lower velocity; and as the velocity increases, the hexagon structure has better and better drag reduction effect. When the load weight is $2.5 \mathrm{~kg}$ and the travel velocity is $0.025 \mathrm{~m} / \mathrm{s}, 6.3 \%$ of the travel drag is reduced.

Due to experimental conditions limit, there is still some problem for further study. This paper mainly studies the phenomenon of resistance distortion and verifies the ship board resistance problem at low velocity. However, the drag reduction effect of bionic ship boards under the rated velocity of general paddy field machinery has not been studied. In the future, exploring wider adaptability drag reduction methods via extending the experiment velocity range will be taken. When studying the bionic structure of the crocodile's armor, just verify the shapes of macroscopic structure has drag reduction effect in the similar dimension, but the structure size parameters optimization for better effect of drag reduction has not be study, so does the ship board materials. In the future, experiments on the interaction between complex multi-medium and micro structure on the ship board will be carried out, which has significant meaning to the bionic drag reduction application on agricultural machinery. In this paper, only two kinds of macroscopic structures are used for experiments, and more parameters (such as ship board area, ship board shapes, 
macroscopic gully structure parameters and so on) will be added in the follow-up study to explore the resistance law under the environment described in this paper. In order to maintain the consistency of each experiment, it takes a lot of time to restore the experiment environment, which is also the main reason why there are not enough samples in this paper, so the experimental method needs to be improved. Because of the complexity of paddy field multi-medium, it is very challenging to establish mathematical model, through more experiment data and analysis, the optimization of drag reduction solutions for specific ship-type paddy field machine is feasible, the establishment of drag reduction model needs further exploration.

\section{Acknowledgements}

The authors gratefully acknowledge the financial support of Guangdong Provincial Special Fund for Modern Agriculture Industry Technology Innovation Teams under Grants No. 2019KJ125; and the fund of Young Innovative Talents Project from Department of Education of Guangdong Province China (Grants No. 2018KQNCX021). The authors wish to thank their generous financial assistance.

\section{[References]}

[1] Yang X B. Analysis of paddy field machinery in China. Agricultural Machinery Market, 2011; 7: 27-30. (in Chinese)

[2] Gameda S, Raghavan G S V, McKyes E, Theriault R. Single and dual probes for soil density measurement. Transactions of the ASAE, 1987; 30(4): 932-944.

[3] Yan G Q. Research on key technologies of light electrical rice transplanter. Guangzhou: South China Agricultural University, 2014. (in Chinese)

[4] Guo Z J, Zhou Z L, Zhang Y, Li Z L. Study on bionic optimum design of soil tillage components. Science in China (Series E: Technological Sciences), 2009; 39(4): 720-728. (in Chinese)

[5] Jia H L, Wang W P, Chen Z, Zheng T Z, Zhang P, Zhuang J. Present situation and prospect of research on optimization of soil-touching parts of agricultural machinery. Transactions of the CSAM, 2017; 48(7): 1-13. (in Chinese)

[6] Wang Z, Li T, Li M, Zhang J Y. Review on drag reduction optimization and mechanism of bionic surface micro structures. Journal of Hebei University of Science and Technology, 2017; 38(4): 325-334. (in Chinese)

[7] Cong Q, Feng Y, Ren L Q. Effect of bionic non-smooth groove shape on drag reduction. Chinese Journal of Hydrodynamics, 2006; 2: 232-238. (in Chinese)

[8] Wu X Q. Study on viscosity reduction and drag reduction characteristics and coupled bionics of earthworm corrugated lubrication body surface. Changchun: Jilin University, 2018. (in Chinese)

[9] Ma Y H, Ma S S, Jia H L, Liu Y C, Peng J, Gao Z H. Measurement and analysis on reducing adhesion and resistance of bionic ripple opener. Transactions of the CSAE, 2014; 30(5): 36-41. (in Chinese)

[10] Ren L Q, Chen D X, Hu J G, Wang L C. Initial exploring for mechanism of decreasing resistance and reducing adhesion of the bionic bulldozing plates. Transactions of the CSAE, 1990; 6(2): 13-20. (in Chinese)

[11] Bixler G D, Bhushan B. Fluid drag reduction with shark-skin riblet inspired microstructured surfaces. Advanced Functional Materials, 2013; 23(36): 4507-4528.

[12] Jia H L, Guo M Z, Zhao J L, Huang D Y, Zhuang J, Qi J T. Design and test of bionic wide-ridge soybean tilling-sowing machine. Int J Agric \& Biol Eng, 2019; 12(1): 42-51.

[13] Jia H L, Wang Q, Huang D Y, Zhu L T, Li M W, Zhao J L. Design of bionic mole forelimb intelligent row cleaners. Int J Agric \& Biol Eng, 2019; 12(3): 27-35.

[14] Zhao J L, Guo M Z, Lu Y, Huang D Y, Zhuang J. Design of bionic locust mouthparts stubble cutting device. Int J Agric \& Biol Eng, 2020; 13(1): 20-28.

[15] Ji Z Y, Lu Z X, Pan J Z. Fuzzy cluster analysis of paddy soil concerning strength and depth of mud layer. Journal of Nanjing Agricultural University, 2000; 1: 101-104. (in Chinese)

[16] Zhang Z W. Operating performance and analysis of floating plate of rice transplanter. Journal of Agricultural Mechanization Research, 1987; 2: 24-28. (in Chinese)

[17] Ren L Q, Chen D X, Chen B C. A summary of study on soil adhesion. Transactions of the CSAE, 1990; 6(1): 1-7. (in Chinese)

[18] $\mathrm{Li} \mathrm{Z} \mathrm{Y,} \mathrm{Wu} \mathrm{W} \mathrm{P.} \mathrm{Discussion} \mathrm{on} \mathrm{reducing} \mathrm{ship} \mathrm{skating} \mathrm{resistance.}$ Transactions of the CSAE, 1988; 4(1): 7-15. (in Chinese)

[19] Fountaine E R. Investigations into the mechanism of soil adhesion. European Journal of Soil Science, 1954; 5(2): 251-263.

[20] Zhao M H, Liu X P, Peng W X. Application of aqueous film theory to study of unsaturated soil's suction. Rock and Soil Mechanics, 2007; 28(7): 1323-1327.

[21] Yan G Q, Zhang T M. A bionic surface drag reduction structure for paddy field ship machinery and its application: China Patent, ZL2014100731933. 2014-06-11. (in Chinese) 